\title{
The Relationship Between Self-Compassion, Concern for Others, and Parental Burnout in Child's Chronic Care Management
}

\author{
Zach Gerber $^{1} \cdot$ Zev Davidovics $^{2} \cdot$ David Anaki ${ }^{1,3}$ (1)
}

Accepted: 7 September 2021 / Published online: 4 October 2021

(c) The Author(s), under exclusive licence to Springer Science+Business Media, LLC, part of Springer Nature 2021

\begin{abstract}
Objectives Parental burnout is a construct that reflects the exhaustion and emotional distancing of parents from their children due to the growing demands of caring for them. Research has pointed to a heightened risk for parental burnout among parents of children with special needs. Additional research has indicated that parents' personality traits and relational dynamics serve as resiliency factors regarding parental burnout.

Methods In the present study, 91 primary parental caregivers of children receiving ongoing treatment at a pediatric ambulatory care unit were recruited. A theoretical model proposing the specific ways in which self-compassion and concern for others complement each other in predicting parental burnout was examined. In this model, the relationships between self-compassion, concern for others, and burnout respectively are mediated by different basic psychological needs, detailed in self-determination theory. Participants completed various questionnaires measuring these constructs. Regression and mediation analyses were used to examine our hypotheses.

Results Results indicated that self-compassion and concern for others predicted levels of parental burnout. The covariance between concern for others and burnout was mediated by the psychological need of relatedness.

Conclusions The findings support the extension of a three-layered conceptual model of (a) self-compassion and concern for others, (b) psychological needs, and (c) burnout. The present study highlights self-compassion and concern for others as potential resilience factors regarding the risk of burnout in parental caregiving. These findings point to promising avenues for burnout prevention and preemptive facilitation of parental caregiving.
\end{abstract}

Keywords Self-compassion $\cdot$ Concern for others $\cdot$ Caregiving $\cdot$ Parental burnout $\cdot$ Self-determination theory

For many, parenthood is one of the most rewarding experiences of their adult life. Yet routinely, parenthood involves demanding challenges, which often result in physical and emotional stress. These tensions sometimes lead to an overall sense of exhaustion. Drawing on the construct of occupational burnout (Maslach et al., 2001), Roskam et al. (2017) coined the term parental burnout (PB) to characterize a similar, yet distinct, form of burnout in parental

David Anaki

david.anaki@biu.ac.il

1 Department of Psychology, Bar-Ilan University, 52900 Ramat Gan, Israel

2 Department of Pediatric Gastroenterology Kiryat Hadassah, Hadassah University Medical Center, 12000 Jerusalem, Israel

3 The Leslie and Susan Gonda (Goldschmied) Multidisciplinary Brain Research Center, Bar-Ilan University, 52900 Ramat Gan, Israel context. Burnout is a unique phenomenon in that it reflects psychological injury resulting primarily from exposure to ongoing stress as opposed to pre-dispositional psychological difficulties. Although PB is considered distinct from psychopathology, it may lead to similar secondary features among parents and children beyond circumstantial stress factors. The consequences of PB are widespread and impact the parent, the spouse/partner, and the child (Mikolajczak, et al., 2018a, b). Specifically, the parent may suffer from addictions, suicidal thoughts, and sleep problems, among other things. In addition, there is a high occurrence of conflicts and estrangements among couples. Finally, the child of a PB parent sometimes suffers from neglect and violence directed toward him.

Like the conceptual structure of occupational burnout, three main features characterized PB (Roskam et al., 2017). Emotional exhaustion is the feeling of being emotionally drained by caring for one's children. The second feature is 
the depletion of personal meaning and satisfaction, which refers to a decline in one's feelings of perceived competence and ability to function successfully as a parent. These two PB features parallel their counterpart features of occupational burnout. The third feature of $\mathrm{PB}$, emotional distancing, differs from its occupational counterpart feature of cynicism and indifference toward others (Roskam et al., 2017). Exhausted employees may objectify their clients or patients by disregarding their subjective individuality. Parents, however, mentally distance themselves from the presence of their children and avoid interacting with them. Preliminary research utilizing network analysis has indicated that this feature of emotional distancing is in fact PB's central feature and central outcome in terms of impacting the child's wellbeing (Blanchard et al., 2021), thereby underscoring PB's distinction from occupational burnout. This distinction has received additional support from Mikolajczak et al. (2020), indicating that each form of burnout is bound to a specific contextual domain (workplace/familial), occurring independent of each other and leading to different consequences.

Roskam et al. (2017) considered PB to vary among all parents in Western societies due to evolving sociodemographic factors that impose accumulating pressure upon families with children, such as the rise in the percentage of households with two working parents. Interestingly, additional research has indicated that parents' personality traits were highly indicative of PB, beyond familial sociodemographic factors and children's particular characteristics (Mikolajczak, et al., 2018a, b). Furthermore, Gérain and Zech (2018) indicated that parents of children with special needs are at a higher risk for developing PB. Moreover, they provide evidence associating the risk for PB primarily with children's daily needs and functional difficulties that require parental attention, beyond the nature of their specific medical condition.

As with PB, occupational burnout is considered a pressing issue among workers in caregiving professions and it has been suggested that a proactive systematic response is necessary (Moss et al., 2016). Recent research has indicated the specific ways in which traits of self-compassion and concern for others complement each other in reducing burnout within a sample of professional caregivers (Gerber \& Anaki, 2020).

Self-compassion entails three main components that overlap and mutually interact: self-kindness, feelings of common humanity, and mindfulness. Self-kindness is the tendency to be caring and understanding of oneself rather than being harshly critical (Neff, 2003a, 2003b). When experiencing difficulty, self-compassionate people embrace themselves and evade self-criticism. The second component, common humanity, entails recognizing that all people fail, make mistakes, and experience pain. A self-compassionate person perceives difficulty and failure as an inevitable part of the shared human experience instead of evidence of his inferiority. When struggling, this feeling of shared experience enables a sense of proximity to others instead of distance. Mindfulness, the third component of self-compassion, reflects one's awareness of the present moment in a clear and balanced manner. Thus, the person neither ignores nor ruminates excessively on troubling aspects of oneself or the environment. Mindfulness entails a broad perspective regarding lived experience beyond transient pain (Brown \& Ryan, 2003). Self-compassion has been associated with an array of negative emotions and overall reduced psychological distress, as well as better coping patterns among parents of children recovering from acute burn injuries (Hawkins et al., 2019; Neff et al., 2018).

Concern for others refers to the positive emotions one feels toward others' welfare, coupled with the motivation to alleviate their sufferings and pains (Strauss et al., 2016). In its healthy form, it combines genuine self-expression with a fundamental perception of the other as having a subjective world of his own (Tolmacz et al., 2019). Concern for others reflects an innate behavioral tendency that appears during childhood and is facilitated by optimal developmental conditions manifested in responding and fulfilling others' needs (Shaver et al., 2019). In adulthood, this behavior is expressed in various forms of caregiving and parenthood (Mikulincer \& Shaver, 2019). Notably, concern for others is associated with secure attachment, reduced psychopathology, and a healthy self-perception (Gerber et al., 2015).

In theory, self-compassion and concern for others are expected to covary due to their shared common humanity factor. However, research has indicated a non-significant correlation between these concepts, and that they reflect the satisfaction of different aspects of well-being (Gerber et al., 2015; Neff \& Pommier, 2013). These findings were expanded in a recent research demonstrating the distinct motivational roles of self-compassion and concern for others regarding caregiver's occupational burnout. This research utilized the theoretical context of self-determination theory (Gerber \& Anaki, 2020).

Self-determination theory (SDT) is a macro-theory of personality, human motivation, and development applied to various domains, including familial settings (Deci \& Ryan, 2000; Ryan \& Deci, 2017). It is considered a unifying framework in the study of personality, motivation, and optimal functioning (Sheldon \& Prentice, 2019). SDT differentiates between two types of motivations: intrinsic, which reflects optimal well-being and functioning over time, and extrinsic, reflecting a lack thereof. Intrinsic motivation consists of acting in line with personal values and interests while experiencing a sense of choice, initiation, and endorsement of one's behavior. Consequently, the main benefits of intrinsically motivated actions are feelings of enjoyment and satisfaction incurred by these behaviors. Conversely, extrinsic motivation reflects the perception of one's actions 
primarily as a means for attaining external rewards. These rewards can be externally controlled, such as avoiding punishment or gaining social approval. These rewards can also be externally autonomous, as in cases where the behavior yields a personally valued outcome. The type of motivation underlying an individual's behavior will determine their psychological well-being and performance over time. Moreover, SDT underscores the crucial role of addressing basic psychological nutriments in upholding intrinsic motivation. These nutriments consist of autonomy, competence, and relatedness. Autonomy is a feeling that one is engaged in an activity stemming from his independent choice and personal values. Competence is a feeling of effectiveness in one's interactions with the natural and social environment. Finally, relatedness is the fulfillment of the need to feel connected and involved with others while experiencing a sense of belonging. Importantly, psychological need satisfaction is considered both foundational to the structuring of one's personality as well as contextually dependent and highly malleable within the familial environment (Sheldon \& Prentice, 2019). Though all three needs are essential, autonomy is primary in facilitating the emergence of competence and relatedness within autonomy-supportive contexts (Weinstein et al., 2010). As for the application of need satisfaction in parenting, thus far, it has been done so primarily regarding strategic need satisfaction of the child (Ryan \& Deci, 2017). However, more recent research has demonstrated how parents' greater psychological need satisfaction facilitates better parenthood in early development and adolescence (Brenning et al., 2019; Nishimura et al., 2020).

In Gerber and Anaki (2020) study of professional caregiving, autonomy mediated the covariance of self-compassion and burnout while relatedness mediated the covariance of concern for others and burnout. The former mediation model was presumably due to autonomy's innate openness to threat, which facilitates constructive feedback when encountering difficulty and limitations (Legault \& Inzlicht, 2013). However, presumably, two reasons may undermine autonomy's mediating role in the particular context of parental caregiving. First, parents do not determine the specific challenges of their parenthood to the same extent as professional caregivers determine the nature of their particular clinical work. Second, parents do not share a professional network or community as professional caregivers do. Therefore, parents often lack the opportunity for an autonomy-supportive context.

Regarding concern for others and PB, parents' concern for others reflects, as in professional caregiving, their desire to fulfill their child's needs. These interpersonal experiences and expectations are considered essential in promoting wellbeing within the familial domain (Ryan \& Deci, 2017). Moreover, its fulfillment nourishes their need for relatedness, as reflected in the association of concern for others with relatedness and multiple other aspects of well-being (Tolmacz et al., 2019).

Drawing on the above, we investigated the role of selfcompassion and concern for others as potential resilience factors against PB among parental caregivers of children with medical conditions that require chronic pediatric ambulatory treatment. We hypothesized that self-compassion and concern for others would each predict reduced PB. Moreover, we hypothesized that self-compassion would be directly associated with PB while concern for others would be indirectly associated with PB through the self-need of relatedness.

\section{Method}

\section{Participants}

Ninety-one parents (76\% woman) of children (mean age $=9.6$ years, $\mathrm{SD}=5.4$ years) who required frequent and ongoing treatment at a single pediatric ambulatory care unit due to a chronic condition participated in the study. Our sample consisted mainly of parents of children with inflammatory bowel disease (IBD) (60\%), as well as other gastroenterology and pulmonary conditions (40\%). Ambulatory care included full-day admissions at least once per month for at least 1 year, during which they received intravenous medication and invasive testing and procedures. Only the primary parental caregiver of each child participated (one parent participant per child) in the current sample. The participants' mean age was 40.3 (range $26-54, \mathrm{SD}=6.2$ ), and the mean age of participant children was 9.6 (range 1-21, $\mathrm{SD}=5.3$ ). The majority of the sample was either married or cohabitating (55\%), while the remaining participants were divorced $(39 \%)$ or single $(6 \%)$. For additional data regarding the association between demographic variables and parental burnout, see Table 1. Ethics approval was obtained from the Helsinki ethics committees of the hospital and from the University Psychology Department.

\section{Procedures}

The first author approached the parents during their child's treatment, presented the research's aim, and requested their participation. Each participant's suitability, namely, that their child required ongoing ambulatory medical care was determined according to the head nurse on site. We did not offer any compensation in exchange for taking part in the study. All consenting participants filled out the questionnaires in their available time during their child's treatment between February 2019 and February 2020. The response rate approximated $76 \%$. The experimenter debriefed the 
Table 1 Zero-sum correlations between parental burnout and demographic variables

\begin{tabular}{|c|c|c|c|c|c|c|}
\hline & PB & Gender & Parent age & Child age & DOT & NOC \\
\hline PB & 1 & & & & & \\
\hline Gender & $-.22 *$ & 1 & & & & \\
\hline Parent age & $-.36^{* *}$ & $.37 * *$ & 1 & & & \\
\hline Child age & $-.24^{*}$ & $.28^{*}$ & $.73 * *$ & 1 & & \\
\hline DOT & -.20 & $.36 * *$ & $.45^{* *}$ & $.55^{* *}$ & 1 & \\
\hline NOC & $-.32^{* *}$ & .03 & $.28 *$ & $.46^{* * *}$ & $.31 *$ & 1 \\
\hline
\end{tabular}

Note: $P B$ parental burnout, $D O T$ duration of treatment, $N O C$ number of children. ${ }^{*} p<.05$, ${ }^{*} p<.01$ participants following the completion and return of the questionnaires.

\section{Measures}

\section{Self-Compassion}

We utilized the 26-item Self-Compassion Scale (SCS, Neff, 2003a). The scale assesses six different aspects of self-compassion (with negative aspects reverse-coded): self-kindness (e.g., "I try to be understanding and patient toward aspects of my personality I don't like"), self-judgment (e.g., “I'm disapproving and judgmental about my own flaws and inadequacies"), common humanity (e.g., "I try to see my failings as part of the human condition"), isolation (e.g., "When I think about my inadequacies it tends to make me feel more separate and cut off from the rest of the world"), mindfulness (e.g., "When something painful happens I try to take a balanced view of the situation"), and over-identification (e.g., "When I'm feeling down I tend to obsess and fixate on everything that's wrong"). Responses are given on a 5-point scale from almost never to almost always. Upon its developments among 391 university undergraduates, the SCS was internally reliable (Cronbach's alpha of 0.92), and demonstrated predictive, convergent, and discriminant validity (Neff, 2003a). The internal consistency of the overall SCS in the present study was 0.88 and we computed an overall SCS average for each participant. Although some have opposed using the total score (Muris \& Petrocchi, 2016; Muris et al., 2016), others have claimed that the factor structure of the SCS supports the use of a total score (Neff, 2016; Neff et al., 2019).

\section{Concern for Others}

We used the empathic concern subscale from the Interpersonal Reactivity Index to measure healthy concern for others (IRI, Davis, 1980). This 8-item subscale evaluates the degree to which the respondent feels warmth, compassion, and concern toward others (e.g., "Seeing warm emotional scenes melts my heart and makes me teary-eyed"). This subscale assesses healthy concern as an emotional component only.
Participants rate the extent to which each item was descriptive of their feelings on a 5-point scale ranging from very not me (1) to very me (5). The IRI was originally developed in a sample of 427 university undergraduates. It has four 7 -item subscales, each of which reflects a different aspect of empathy (empathic concern, perspective taking, personal distress, and fantasy). The internal reliabilities of the subscales ranged from 0.71 to 0.77 and the test-retest reliabilities from 0.61 to 0.71 . Various studies have determined the IRI's convergent and external validity, as well as its 4-factor structure (Fernández et al., 2011; Pulos et al., 2004). In the current study, Cronbach's alpha was 0.81 .

\section{Basic Psychological Needs Scale (BPNS, Gagné, 2003)}

The scale was adapted from a measure of need satisfaction at work (Ilardi et al., 1993). The BPNS consists of separate subscales measuring from a trait perspective the satisfaction levels of the need for autonomy ("I feel like I can pretty much be myself in my daily situations"), competence ("Most days I feel a sense of accomplishment from what I do"), and relatedness ("I consider the people I regularly interact with to be my friends"). The internal reliability was $0.69,0.86$, and 0.71 for the autonomy, relatedness, and competence subscales, respectively. In the present study, Cronbach's alpha was $0.70,0.77$, and 0.59 .

\section{Parental Burnout Inventory (PBI; Roskam et al., 2017)}

We utilized the PBI to measure burnout among parents serving as primary informal caregivers to a child with a severe medical condition that requires extended ambulatory care. The scale has 22 items answered on a 6-point Likert scale and indexes the cumulative effects of parental-related stressors on the three dimensions of (a) emotional distance ("I can no longer show my children how much I love them"), (b) emotional exhaustion ("I am at the end of my patience at the end of a day with my children"), and (c) sense of personal accomplishment ("I am easily able to create a relaxed atmosphere with my children"). Higher scores for the first two scales and lower scores for the last one are indicative of burnout. Upon its developments among 1723 parents, the 
PBI was internally reliable (Cronbach's alpha ranged from 0.87 to 0.95 across the three factors), and demonstrated predictive, convergent, and discriminant validity (Roskam et al., 2017). In the current sample, the overall Cronbach's alpha was 0.89 .

\section{Data Analyses}

Data were examined for normality, multicollinearity, and the presence of outliers or impossible values. Missing values were minimal and were excluded pairwise from analysis $(\sim 1.5 \%)$. Participant demographic characteristics, zero-order correlations, and hierarchical regression were calculated with the SPSS 22 software. Due to the sample size, a SEM path analysis approach that enables the examination of simultaneous mediation models could not be implemented. Therefore, mediation effects were examined using the PROCESS software (for SPSS Release 3.00; Hayes, 2017). The overall regression model was also limited to five predictors, as determined by an a priori power analysis (G*Power, Faul et al., 2007).

\section{Results}

In order to estimate the unmediated relations among the variables of interest, we conducted a zero-sum correlation analysis (Table 2). Notably, self-compassion and concern for others were unrelated $(r=0.02, p>0.88)$. Self-compassion was correlated with autonomy $(r=0.40, p<0.0001)$ and competence ( $r=0.44, p<0.0001)$, while concern for others was correlated with relatedness $(r=0.46, p<0.0001)$. PB was negatively correlated with both concern for others $(r=-0.31, p<0.004)$ and self-compassion $(r=-0.38$, $p<0.0001)$. PB was also negatively correlated with all three psychological need satisfactions, namely autonomy, competence, and relatedness (range $=-0.33$ to $-0.47, p s<0.001$ ).

We then conducted a two-step hierarchical regression analysis with parental burnout as the outcome variable to address our hypothesis. In the first step of the analysis, we entered participants' self-compassion and concern for others to assess their independent PB predictions. In the second step, we added the psychological needs measures of autonomy, relatedness, and competence. This step allowed us to examine the potential mediating roles of self-needs regarding the contribution of self-compassion and concern for others to PB.

The first step was significant, $F(2,86)=13.00, p<0.0001$, $R^{2}=0.24$, with both self-compassion and concern for others making a significant contribution $(\beta=-0.38, p<0.0001$, and $\beta=-0.30, p<0.002$, respectively, Table 3 ). These findings support our first hypothesis that self-compassion and concern for others are complimentary in predicting PB levels. In the second step, the addition of the self-needs scores yielded a significant change in the variance accounted for, $\Delta F(5,86)=9.97, p>0.0001, \Delta R^{2}=0.15$, with relatedness making a significant contribution $(\beta=-0.33, p>0.004)$. Autonomy and competence, however, were not significant ( $\beta=-0.21, p=0.05$, and $\beta=0.04, p>0.70$, respectively). In addition, self-compassion remained significant while concern for others did not after the self-needs scores were added to the set of predictors $(\beta=-0.28, p<0.007$, and $\beta=-0.14, p=0.17$, respectively). These latter results support our hypothesis that the contribution of self-compassion to PB is not mediated by autonomy or the other psychological need satisfactions. They also support our hypothesis that the relationship between concern for others and PB is mediated by the relatedness self-need.

Table 3 (Un)standardized regression coefficients predicting parental burnout

\begin{tabular}{llll}
\hline & & \multicolumn{2}{c}{ Parental burnout } \\
\cline { 3 - 4 } & Variable & $B$ & $\beta$ \\
\hline Step 1 & Self-compassion & -.50 & $-.38^{* * *}$ \\
& Concern for others & -.46 & $.30^{* * *}$ \\
Step 2 & Self-compassion & -.38 & $-.28^{* *}$ \\
& Concern for others & -.21 & -.14 \\
& Autonomy & -.18 & -.21 \\
& Relatedness & -.33 & $-.33^{* *}$ \\
& Competence & .04 & .04 \\
\hline
\end{tabular}

${ }^{*} p<.05, * * p<.01, * * * p<.001$
Table 2 Zero-sum correlations between all variables in regression model and path analysis

\begin{tabular}{lllllll}
\hline & PB & SC & Concern & Autonomy & Relatedness & Competence \\
\hline PB & 1 & & & & & \\
SC & $-.38^{* *}$ & 1 & & & \\
Concern & $-.31^{* *}$ & .02 & 1 & & \\
Autonomy & $-.43^{* *}$ & $.40^{* *}$ & .10 & 1 & \\
Relatedness & $-.47^{* *}$ & .10 & $.46^{* *}$ & $.34 * *$ & 1 & \\
Competence & $-.33^{* *}$ & $.44^{* *}$ & .08 & $.44 * *$ & $.44 * *$ & 1 \\
\hline
\end{tabular}

Note: $P B$ parental burnout, $S C$ self-compassion, Concern concern for others. $* p<.05, * * p<.01$ 
Finally, we examined the potential mediation of relatedness on the relationship between concern for others and PB using the PROCESS software. As Fig. 1 illustrates, the standardized regression coefficient between concern for others and relatedness was statistically significant, as was the standardized regression coefficient between concern for others and PB. The standardized indirect effect was -0.28 $(0.70 \times-0.40)$ and it fully mediated the association between concern for others and PB. We tested the significance of this indirect effect using bootstrapping procedures. Unstandardized indirect effects were computed for each of 10,000 bootstrapped samples, and the $95 \%$ confidence interval was computed by determining the indirect effects between the 2.5 th and 97.5 th percentiles. The bootstrapped unstandardized indirect effect was -0.28 , and the $95 \%$ confidence interval ranged from -0.48 to -0.11 . Thus, the indirect effect was statistically significant. Importantly, we replicated the analysis and confirmed its results while controlling for the demographic variables associated with parental burnoutparent age, gender, number of children, and the age of child in need of medical treatment, as presented in Table $1(95 \%$ CI: -0.58 to -0.12 ). Furthermore, there was no evidence that concern for others influenced burnout indirectly through autonomy (95\% CI: -0.22 to 0.06$)$ or competence $(95 \%$ CI: -0.15 to 0.05$)$.

\section{Discussion}

The present study examined the role of self-compassion and concern for others in parental burnout (PB) among parents of children in need of ongoing pediatric ambulatory care. We collected data, utilizing multiple self-report measures, and fitted them into a predetermined theoretical mediation model. Our primary goal was to extend the generalizability of similar research recently conducted among professional caregivers (Gerber \& Anaki, 2020). The present study's three notable findings are as follows: first, selfcompassion and concern for others each predicted PB levels. Second, autonomy did not mediate the covariance between

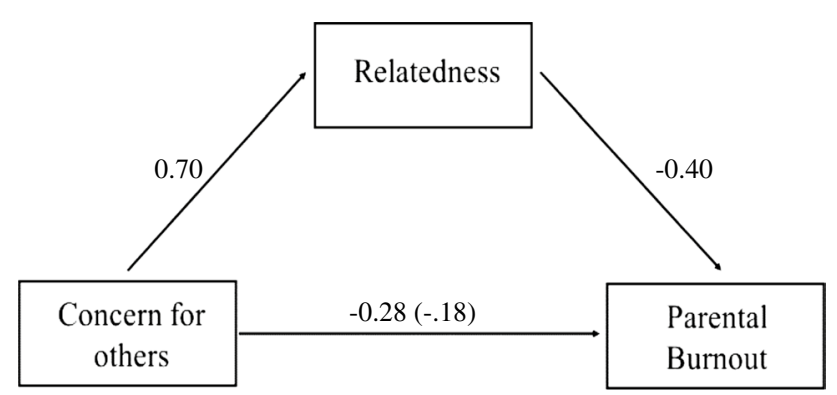

Fig. 1 Standardized coefficients of the mediation model self-compassion and PB. Finally, relatedness mediated the covariance between concern for others and PB.

Our first finding that self-compassion and concern for others did not share any covariance with PB converges with previous results (Gerber \& Anaki, 2020; Gerber et al., 2015). These findings indicate that self-compassion and concern for others are distinct yet complementary constructs in their contribution to reduced burnout among caregivers. Moreover, it underscores the importance of directly addressing parental needs, apart and independently of their child's perceived necessities, to enhance parenthood quality. Notably, $\mathrm{PB}$ reflects a reduction in parental well-being as well as in parental performance and responsiveness. Therefore, directly addressing parental needs is a valuable way of improving the child's quality of parenthood (Jacobs \& Wachs, 2002; Roskam et al., 2017). Given that PB is particularly relevant regarding parents of children with an ongoing medical condition, coping with it should be addressed directly as part of their overall medical care (Gérain \& Zech, 2018). Indeed, proactively building parental resilience by actively cultivating parental self-compassion is a promising avenue for further pediatric clinical intervention (Brassington \& Lomas, 2020; Germer \& Neff, 2019).

The role of self-compassion regarding PB among parental caregivers differs from its relationship to burnout among professional caregivers. While we found a direct association between self-compassion and PB, this association was mediated through the self-need fulfillment of autonomy among professional caregivers. We anticipated this given that, presumably, parental caregivers do not experience autonomy in the context of their caregiving in the same manner professional caregivers do. Interestingly, the remaining two selfneeds, namely competence and relatedness, did not mediate self-compassion and PB covariance. This pattern suggests that self-compassion contributes to parental caregiving in a way that is not motivational per se. Berman (2014) characterized parenthood as an innate and insurmountable thrust to overcome what is perceived as a source of harm or pain to their child. We presume the direct association between self-compassion and PB reflects a parental acknowledgment of their existential limitations in fulfilling this thrust. This parental recognition is constructive to their perception of parenthood. Notably, self-compassion in and of itself is a way of self-soothing, especially when struggling with a harsh experience (Germer, 2009).

Indeed, parents' efforts to be present alongside their children, struggling with a severe medical condition, familiarizes them with their limitations in preventing their child from experiencing pain. By attempting to be present and responsive to their child when most vulnerable, parents are exposed to the existential limits of their parental abilities in preventing their child from experiencing pain. Presumably, when existential limitations are acknowledged through 
a self-compassionate perspective, parents are better able to remain present on behalf of their child who needs their support. In contrast, a lack of self-compassion has been found to facilitate withdrawal from aversive situations and avoid its facts (Gerber \& Anaki, 2019).

Drawing on this understanding, we turn to our third finding regarding the dynamics between concern for others and PB. In contrast to the direct association between self-compassion and $\mathrm{PB}$, concern for others relates to $\mathrm{PB}$ through the psychological nutriment of relatedness. As mentioned, recent research indicates that the primary feature of PB is emotional distance, which purportedly reflects a lack of relatedness (Blanchard et al. 2021). Seemingly, it is the parent's relationship with their child and the time spent together that is most fulfilling to the former in their parenthood endeavor. Our finding that relatedness mediated the covariance between concern for others and PB suggests that for a parental caregiver, the relationship in and of itself with their child in need is rewarding and salutogenic from an SDT perspective. From a clinical point of view, this finding suggests that parents should be encouraged to express concern and personally relate to their child in need to improve their parental well-being.

\section{Limitations and Future Research}

The present study has several limitations. First, the crosssectional design prevents us from making any causal inferences and undermines our proposed theoretical directionality. Given that our findings provide only a snapshot of a permanent reality longitudinal research is highly relevant. Continuous measures over time are especially warranted, given the meandering dynamic of complex pediatric medical conditions. Second, our research sample was considerably small, limiting a rigorous conclusion of the statistical analyses we applied. Moreover, the data collected was based on participants' self-report measures and are therefore subject to confirmation and self-serving biases. In addition, some of the findings in our study may suffer from common methods bias in which observed measures covariance reflects their common mode of self-report measurement beyond their conceptual association at the construct level (Podsakoff, et al., 2012). In a recent study, PB was linked to a stress measure of skin cortisol concentration, which may provide a practical biological marker of PB in future research (Brianda et al., 2020).

The importance of PB cannot be underestimated, both under normal circumstances but especially in stressful conditions such as the era of COVID-19 (Mikolajczak \& Roskam, 2020). However, PB research is in its infancy, and additional studies are required to elucidate the factors that facilitate it and determine the clinically significant PB levels in clinical intervention. In addition, given that our sample consisted mainly of women, future research should examine whether the present findings are generalizable to men (Roskam \& Mikolajczak, 2020).

Finally, although our study focused on parental caregivers of chronically ill children in need of ambulatory care, our results are also relevant for other types of informal caregivers and parents of typical healthy children (Adashek \& Subbiah, 2020; Gérain \& Zech, 2018). For example, future studies could investigate the current theoretical model among spouses of adults coping with a chronic debilitating medical condition. In addition, future research could examine our theoretical model among a sample of healthy children's parents. This examination will allow exploring whether the parental burnout resilience factors identified in the current study can be applied to parenthood in general.

Supplementary Information The online version contains supplementary material available at https://doi.org/10.1007/s12671-021-01752-z.

Author Contribution ZG designed and executed the study, assisted with the data analyses, and wrote the paper. ZD assisted with participants' recruitment and in writing and editing the manuscript. DA collaborated with the study's design, analyzing the data, and writing the manuscript. All authors collaborated in the writing and editing of the final manuscript.

Data Availability All data are available at Supplementary Materials.

\section{Declarations}

Ethical Approval The study was approved by the Helsinki ethics committees of Hadassah University Medical Center (Jerusalem, Israel), and by the Psychology Department at Bar-Ilan University (Ramat Gan, Israel).

Informed Consent All participants signed informed consent before participating in the study.

\section{References}

Adashek, J. J., \& Subbiah, I. M. (2020). Caring for the caregiver: A systematic review characterizing the experience of caregivers of older adults with advanced cancers. ESMO Open, 5, e000862. https://doi.org/10.1136/esmoopen-2020-000862

Berman, E. (2014). The Happy Prince, The Giving Tree: The fantasy of parenthood as self-annihilation and its relevance to psychoanalytic treatment. Relational Psychoanalysis, 4, 159-176.

Blanchard, M. A., Roskam, I., Mikolajczak, M., \& Heeren, A. (2021). A network approach to parental burnout. Child Abuse Neglect, 111, 104826. https://doi.org/10.31234/osf.io/swqfz

Brassington, K., \& Lomas, T. (2020). Can resilience training improve well-being for people in high-risk occupations? A systematic review through a multidimensional lens. The Journal of Positive Psychology, 1-20. https://doi.org/10.1080/17439760.2020.17527 83

Brenning, K., Soenens, B., Mabbe, E., \& Vansteenkiste, M. (2019). Ups and downs in the joy of motherhood: Maternal well-being 
as a function of psychological needs, personality, and infant temperament. Journal of Happiness Studies, 20, 229-250.

Brianda, M. E., Roskam, I., \& Mikolajczak, M. (2020). Hair cortisol concentration as a biomarker of parental burnout. Psychoneuroendocrinology, 117, 1-5.

Brown, K. W., \& Ryan, R. M. (2003). The benefits of being present: Mindfulness and its role in psychological well-being. Journal of Personality and Social Psychology, 84, 822-848.

Davis, M. H. (1980). A multidimensional approach to individual differences in empathy. Catalog of Selected Documents in Psychology, 10, 1-17.

Deci, E. L., \& Ryan, R. M. (2000). The "what" and "why" of goal pursuits: Human needs and the self-determination of behavior. Psychological Inquiry, 11, 227-268.

Faul, F., Erdfelder, E., Lang, A. G., \& Buchner, A. (2007). G* Power 3: A flexible statistical power analysis program for the social, behavioral, and biomedical sciences. Behavior Research Methods, 39, 175-191.

Fernández, A. M., Dufey, M., \& Kramp, U. (2011). Testing the psychometric properties of the Interpersonal Reactivity Index (IRI) in Chile. European Journal of Psychological Assessment, 27, $179-185$.

Gagné, M. (2003). The role of autonomy support and autonomy orientation in prosocial behavior engagement. Motivation and Emotion, 27(3), 199-223.

Gerber, Z., \& Anaki, D. (2020). The role of self-compassion, concern for others, and basic psychological needs in the reduction of caregiving burnout. Mindfulness.

Gerber, Z., \& Anaki, D. (2019). Self-compassion as a buffer against concrete but not abstract threat. Death Studies, 43, 639-646.

Gerber, Z., Tolmacz, R., \& Doron, Y. (2015). Self-compassion and forms of concern for others. Personality and Individual Differences, 86, 394-400.

Gérain, P., \& Zech, E. (2018). Does informal caregiving lead to parental burnout? Comparing parents having (or not) children with mental and physical issues. Frontiers in Psychology, 9, $1-10$.

Germer, C., \& Neff, K. (2019). Teaching the mindful self-compassion program: A guide for professionals. Guilford Publications.

Germer, C. (2009). The mindful path to self-compassion: Freeing yourself from destructive thoughts and emotions. Guilford Press.

Hawkins, L., Centifanti, L. C., Holman, N., \& Taylor, P. (2019). Parental adjustment following pediatric burn injury: The role of guilt, shame, and self-compassion. Journal of Pediatric Psychology, 44, 229-237.

Hayes, A. F. (2017). Partial, conditional, and moderated moderated mediation: Quantification, inference, and interpretation. Сommunication Monographs, 85(1), 4-40

Ilardi, B. C., Leone, D., Kasser, T., \& Ryan, R. M. (1993). Employee and supervisor ratings of motivation: Main effects and discrepancies associated with job satisfaction and adjustment in a factory setting. Journal of Applied Social Psychology, 23, 1789-1805.

Jacobs, L., \& Wachs, C. (2002). Parent therapy: A relational alternative to working with children. Jason Aronson Inc.

Legault, L., \& Inzlicht, M. (2013). Self-determination, self-regulation, and the brain: Autonomy improves performance by enhancing neuroaffective responsiveness to self-regulation failure. Journal of Personality and Social Psychology, 105, 123-138.

Maslach, C., Schaufeli, W. B., \& Leiter, M. P. (2001). Job burnout. Annual Review of Psychology, 52, 397-422.

Mikolajczak, M., Raes, M. E., Avalosse, H., \& Roskam, I. (2018a). Exhausted parents: Sociodemographic, child-related, parentrelated, parenting and family-functioning correlates of parental burnout. Journal of Child and Family Studies, 27, 602-614.

Mikolajczak, M., Gross, J. J., Stinglhamber, F., Norberg, A. L., \& Roskam, I. (2020). Is parental burnout distinct from job burnout and depressive symptomatology? Clinical Psychological Science, 8, 673-689.

Mikolajczak, M., Brianda, M. E., Avalosse, H., \& Roskam, I. (2018b). Consequences of parental burnout: Its specific effect on child neglect and violence. Child Abuse \& Neglect, 80, 134-145.

Mikolajczak, M., \& Roskam, I. (2020). Parental burnout: Moving the focus from children to parents. New Directions for Child and Adolescent Development, $1-7$.

Mikulincer, M., \& Shaver, P. R. (2019). Attachment, caregiving, and parenting. In O. Taubman - Ben-Ari (Ed.), Pathways and barriers to parenthood. Springer.

Moss, M., Good, V. S., Gozal, D., Kleinpell, R., \& Sessler, C. N. (2016). An official critical care societies collaborative statement: Burnout syndrome in critical care health care professionals: A call for action. American Journal of Critical Care, 25, 368-376.

Muris, P., Otgaar, H., \& Petrocchi, N. (2016). Protection as the mirror image of psychopathology: Further critical notes on the SelfCompassion Scale. Mindfulness, 7, 787-790.

Muris, P., \& Petrocchi, N. (2016). Protection or vulnerability? A meta- analysis of the relations between the positive and negative components of self-compassion and psychopathology. Clinical Psychology and Psychotherapy, 24, 373-383.

Neff, K. D. (2003a). Development and validation of a scale to measure self-compassion. Self and Identity, 2, 223-250.

Neff, K. D. (2003b). Self-compassion: An alternative conceptualization of a healthy attitude toward oneself. Self and Identity, 2, 85-102.

Neff, K. D. (2016). The self-compassion scale is a valid and theoretically coherent measure of self-compassion. Mindfulness, 7 , 264-274.

Neff, K. D., Long, P., Knox, M., Davidson, O., Kuchar, A., Costigan, A., Williamson, Z., Rohleder, N., Tóth-Király, I., \& Breines, J. G. (2018). The forest and the trees: Examining the association of self-compassion and its positive and negative components with psychological functioning. Self and Identity., 17, 627-645.

Neff, K. D., \& Pommier, E. (2013). The relationship between self-compassion and other-focused concern among college undergraduates, community adults, and practicing meditators. Self and Identity, 12(2), 160-176

Neff, K. D., Tóth-Király, I., Yarnell, L. M., Arimitsu, K., Castilho, P., Ghorbani, N., Guo, H. X., Hirsch, J. K., Hupfeld, J., Hutz, C. S., Kotsou, I., Lee, W. K., Montero-Marin, J., Sirois, F. M., de Souza, L. K., Svendsen, J. L., Wilkinson, R. B., \& Mantzios, M. (2019). Examining the factor structure of the Self-Compassion Scale in 20 diverse samples: Support for use of a total score and six subscale scores. Psychological Assessment, 31, 27-45.

Nishimura, T., Bradshaw, E. L., Deci, E. L., \& Ryan, R. (2020). Satisfaction of basic psychological needs in an interdependence model of fathers' own aspirations and those of their adolescent children. Social Development, 30, 293-310.

Podsakoff, P. M., MacKenzie, S. B., \& Podsakoff, N. P. (2012). Sources of method bias in social science research and recommendations on how to control it. Annual Review of Psychology, 63, 539-569.

Pulos, S., Elison, J., \& Lennon, R. (2004). Hierarchical structure of the Interpersonal Reactivity Index. Social Behavior and Personality, $32,355-360$.

Roskam, I., \& Mikolajczak, M. (2020). Gender differences in the nature, antecedents and consequences of parental burnout. Sex Roles, 83, 485-498.

Roskam, I., Raes, M. E., \& Mikolajczak, M. (2017). Exhausted parents: Development and preliminary validation of the parental burnout inventory. Frontiers in Psychology, 8, 1-12.

Ryan, R. M., \& Deci, E. L. (2017). Self-determination theory: Basic psychological needs in motivation, development, and wellness. Guilford Publications. 
Shaver, P. R., Mikulincer, M., \& Cassidy, J. (2019). Attachment, caregiving in couple relationships, and prosocial behavior in the wider world. Current Opinion in Psychology, 25, 16-20.

Sheldon, K. M., \& Prentice, M. (2019). Self-determination theory as a foundation for personality researchers. Journal of Personality, $87,5-14$.

Strauss, C., Taylor, B. L., Gu, J., Kuyken, W., Baer, R., Jones, F., \& Cavanagh, K. (2016). What is compassion and how can we measure it? A review of definitions and measures. Clinical Psychology Review, 47, 15-27.

Tolmacz, R., Friedemann, Y., Doron, Y., \& Gerber, Z. (2019). Narcissism and concern for others: A contradiction in terms? Current Psychology, 1, 1-10.
Weinstein, N., Hodgins, H. S., \& Ryan, R. M. (2010). Autonomy and control in dyads: Effects on interaction quality and joint creative performance. Personality and Social Psychology Bulletin, 36, $1603-1617$.

Publisher's Note Springer Nature remains neutral with regard to jurisdictional claims in published maps and institutional affiliations. 